\title{
Better Early Packet Discard
}

\author{
Hajer Tounsi Naouar, Farouk Kamoun \\ Ecole Nationale des Sciences de l'Informatique, campus universitaire de Manouba, 2010 \\ Manouba, TUNISIE. Hajer.Tounsi@ensi.rnu.tn, Farouk.Kamoun@ensi.rnu.tn
}

\begin{abstract}
Multimedia Applications require more control at the application level as well as the network level to minimize the negative impact of the single Best Effort service offered in the Internet. The deployment of Active queue management is recommended to react to congestion before it becomes critical.

In this paper, we propose and compare some variants of EPD (Early Packet Discard), named BEPD and EPD-3 for the transmission of MPEG video streams over the Internet. We have shown by simulation that EPD, BEPD and EPD-3 increase significantly the number of transmitted I frames in comparison to the "Tail Drop" technique. BEPD improves the performance of video traffic by accepting in the queue only pictures that their reference ones have already been accepted in the queue. EPD-3 gives better results than EPD and BEPD by dropping first $B$ pictures, then $P$ pictures and finally I pictures.

Assured forwarding service proposed by DiffServ defines 4 independent forwarding classes. Within each of these classes, there are 3 levels of delivery which can be used to introduce some loss priority between different types of MPEG video Pictures. However, this information is not sufficient to use with EPD and its variants. We need more information about the semantic of packets. We propose and specify an extension that describes the type and the position of each packet in the video stream.
\end{abstract}

Keywords : $\quad$ QoS, Buffer management, Tail Drop, EPD, MPEG.

The original version of this chapter was revised: The copyright line was incorrect. This has been corrected. The Erratum to this chapter is available at DOI: 10.1007/978-0-387-35620-4_43 


\section{INTRODUCTION}

Over time, the needs and uses of the Internet have changed. The Internet is becoming a medium for business, learning and entertainment communications which need several types of media such as voice and video.

Currently, the Internet offers a single "Best Effort" service that is not adequate for multimedia traffic because it doesn't offer any quality of service guarantees. Congestion Control mechanisms, such as slow start, and congestion avoidance algorithms implemented with TCP are not adequate to control video and audio traffic [RFC 2001]. To transport multimedia traffic over the Internet, some different control mechanisms should be added in the network.

Different approaches (IntServ, DiffServ, MPLS) are proposed by the IETF researchers to overcome this problem. They are not necessarily mutually exclusive.

In IntServ (Integrated Services) approach [RFC 1633], the network offers different classes of service (Controlled load, Guaranteed service) [RFC 2211] [RFC 2212] according to the applications requirements. The IntServ architecture defines preventive control mechanisms such as admission control, classification, scheduling and signalling mechanisms. Several studies have shown that the use of the RSVP signalling mechanism (Resource reSerVation Protocol) [RFC 2205] in the Internet is not scalable. This is mainly due to the complexity involved in managing the flow states in Internet backbone routers and the lack of mechanisms allowing users to reserve needed resources without under-utilizing the network. Another main obstacle to implementing this model is due to the pricing scheme of several services with different traffic parameters.

The Differentiated Services (DiffServ) approach, is proposed to provide scalable service discrimination in the Internet without the need of per-flow state and signalling at every hop [RFC 2475]. Scalability is achieved by implementing complex conditioning functions (classification, marking, shaping) at network edge nodes, and by applying per-hop behaviour to flows which have been marked using the DSCP (Differentiated Service Code Point) field in IPv4 or IPv6 headers.

The differentiation between flows or set of flows requires that the network controls the amount of resources that each flow or set of flows is allowed to consume. Scheduling mechanisms, such as variants of Round Robin and Fair Queuing supply fairness by isolating different flows or classes of flows. They are used primarily to manage the allocation of bandwidth among a set of flows or classes and prevent some traffic, such as multimedia flows, from exceeding their share of bandwidth in periods of congestion. But these per-flow algorithms do nothing to control the overall 
queue size or to smooth the dropping of packets which is the matter of buffer queue management schemes.

With the increasing interest of transmitting video streams over Internet and especially the MPEG video streams, efficient video-oriented dropping schemes have to be designed which attempt to control picture quality degradation during network congestion.

In [TOU 98] [TOU 99], we have proposed the deployment of EPD (Early Packet Discard) mechanism for the transmission of MPEG1/2 video streams. EPD consists in discarding all the packets of one application data unit, taken as a MPEG GOP (Group of Picture), if one of its packets is dropped. We have shown by simulation that EPD improves the performance of video traffic by accepting more GOPs than Tail Drop.

In this paper, we take the MPEG picture as a data unit for the deployment of EPD (Early Packet Discard) mechanism. We show by simulation that EPD is better than the traditional buffer management "Tail Drop". We propose and compare other variants of EPD referred as BEPD and EPD-3, which take account of the hierarchy structure of MPEG and the dependence of its components.

The paper is organized as follows. In section 2, we briefly review the structure of MPEG1/2 video streams, and show the motivation to use an active queue management mechanism such as EPD. Section 3 describes the techniques which we propose to be used for MPEG video transmission and presents the performance results of these schemes. Section 4 specifies the multimedia option that we propose to inform EPD routers about traffic characteristics. Finally, we conclude in section 5.

\section{MPEG CHARACTERISTICS}

In this paper we are interested in the transmission of MPEG video streams. MPEG defines a video stream as a hierarchy of data structures : block, macro-block, slice, frame or picture, group of pictures (Figure 1).

A frame or a picture is the basic unit of display. Three types of pictures are defined in MPEG video stream. They differ from the encoding method used: Intra-coded picture (I), Predictive-coded picture (P) and Bidirectionally predictive-coded picture (B)(Figure 2 ). I and $\mathrm{P}$ pictures are reference pictures so they are essential and have to be preserved from corruption during transmission, otherwise the quality of pictures will be degraded. I-frame corruption affect all subsequent frames in the same Group of pictures (GOP). Similarly, the impairment of P-frames affect all following $P$ and $B$ frames. Only B-frame impairments have no effect on other frames. 
According to the above, it should be noted that in situation of congestion, dropping video packets indiscriminately can cause serious degradation in picture quality. The transmission of some packets can be wasteful if they are related to other packets which have been dropped. Intra and predictive pictures have to be better protected from loss during transmission. Thus, it is critical to choose a dropping policy that ensures the best performance for video traffic.

\begin{tabular}{|l|l|l|}
\hline Sequence header & GOP & GOP \\
\hline GOP header & Picture & Picture \\
\hline Picture header & Slice & Slice \\
\hline Slice header & Macrobloc & Macrobloc \\
\hline Macrobloc & Bloc & Bloc \\
\hline
\end{tabular}

Figure 1. MPEG1 video hierarchy

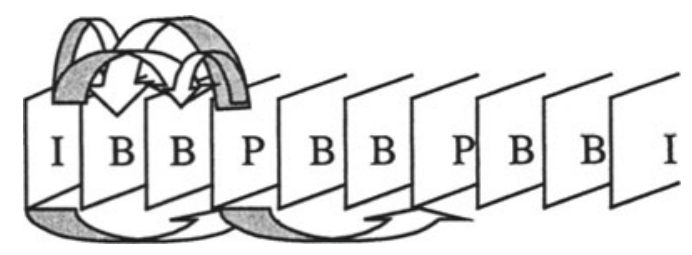

Figure2. MPEG frames dependence

\section{PERFORMANCE EVALUATION OF BUFFER MANAGEMENT SCHEMES}

The traditional technique for managing router queue lengths, known as "Tail drop" consists of setting a maximum length for each queue, accepting packets for the queue until the maximum length is reached, then dropping the next packets until the queue size decreases. This technique allows queues 
to maintain a full status for long periods, and causes the drop of many packets when they arrive in bursts. In the case of MPEG video, the first incoming pictures can fit the queue, after that the queue becomes full and some packets of pictures are dropped. Some useless packets occupy the queue space preventing other packets from getting room in the queue.

To cope with the frame fragmentation and poor transmission performance of the traditional Tail Drop technique, we deploy Early packet Discard (EPD) for the transmission of MPEG video [TOU 99], and we propose some variants of EPD to improve the quality of the MPEG video stream by maximizing the number of transmitted Intra frames.

\subsection{Early Packet Discard (EPD)}

This mechanism is a variant of the Partial Packet Discard mechanism (PPD) proposed in [ROM 95]. PPD is defined to improve the throughput performance of the TCP over ATM. In fact, the analysis of the throughput behaviour shows that the poor performance of TCP over ATM is caused by the problem of fragmentation of the TCP packets in ATM cells and the random drop of ATM cells in case of congestion. So, in PPD if a cell is dropped by a switch, the subsequent cells are also dropped.

In Early Packet Discard, when the switch buffer queue reaches a threshold level, entire newly arriving packets (AAL5 PDU) are discarded[ROM 95] rather than only a portion of them as it may happen in PPD.

[MEH 98] proposes some enhancements to PPD and EPD in order to cope with the problem of packet fragmentation for the transmission of MPEG video streams over ATM. The proposed scheme, named Adaptive early video slice discard (A-ESD), consists in dropping entire video slices prior to congestion and gives priority to the $\mathrm{I}$ and $\mathrm{P}$ cells.

In [TOU 98] [TOU 99], we have proposed the deployment of EPD for the transmission of MPEG video over Internet. We have shown that EPD is better than Tail Drop by operating on the similarity between a TCP packet fragmented in ATM cells and a MPEG GOP (Group of Pictures) fragmented in IP packets. When the threshold level is reached, the router accepts packets included in a GOP already inserted, and drops the next GOPs. We have shown that EPD maximizes the number of entire GOPs reaching the destination.

In this paper, we implement EPD with a data unit taken as a picture. In fact, the picture is the basic unit of display and its importance depends on its type. The most important ones are I frames.

\subsection{Performance Evaluation of EPD}




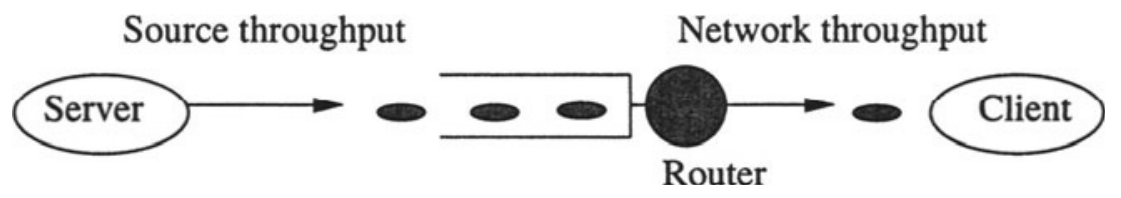

Figure 3. Simulation Scenario

We choose a simple scenario made up of three nodes: a server (sender of the MPEG video flow), a congested edge router and a client (receiver of the flow)(Figure 3). The simulations are run in the case of a saturated system illustrated by taking a network throughput much less than the source throughput and a limited node buffer size. The network behaviour is modelled by the network rate. The simulation manipulates many parameters, such as the packet length, the source throughput, the queue length, the threshold for EPD and the network bandwidth. The input MPEG video flows, extracted from real video sequences, are coded with a fixed number of pictures per second $(25 \mathrm{p} / \mathrm{s})$. The pictures have different lengths. One picture is composed of a variable number of packets. We choose for each packet a fixed arbitrary length equals to 1000 bytes.

We run simulations for a network throughput equals to $512 \mathrm{~kb} / \mathrm{s}$ and a buffer size equals to 100 kbytes. We take a EPD threshold equals to half of the buffer (50 kbytes) as recommended in [TUR 96]. Different MPEG-1 video traces (movie sequence, sport events, Interview) have been tested. We have noted in all cases that EPD gives better results than Tail Drop.

In the following, we present the results corresponding to the transmission of an Interview video sequence (Table1).

\begin{tabular}{|c|c|c|c|c|}
\hline Sequence & $\begin{array}{c}\text { GOP pattern } \\
\text { (12 frames) }\end{array}$ & $\begin{array}{c}\text { I frame } \\
\text { Average size } \\
\text { (bytes) }\end{array}$ & $\begin{array}{c}\text { P frame } \\
\text { average size } \\
\text { (bytes) }\end{array}$ & $\begin{array}{c}\text { B frame } \\
\text { average size } \\
\text { (bytes) }\end{array}$ \\
\hline Inteview & IBBPBBP ... & 20396 & 5564 & 2947 \\
\hline
\end{tabular}

Table 1 : Characteristics of the Interview sequence

Figure 4 shows the picture success rate, defined as the number of received pictures vs the the total transmitted pictures, in function of their type. We note that Tail Drop allows the transmission of a small number of I and $P$ frames, but almost $50 \%$ of $B$ frames (47.3\% for this particular flow). This leads to a bad quality of the stream, because B pictures couldn't be decoded without their reference frames. However, EPD allows the transmission of a greater number of reference pictures $(84.3 \%$ of I frames and $38.8 \%$ of $P$ frames for this particular flow) than Tail Drop, but a small number of transmitted B-frames (28\% for this particular flow). So, EPD assure a better quality than Tail Drop. 


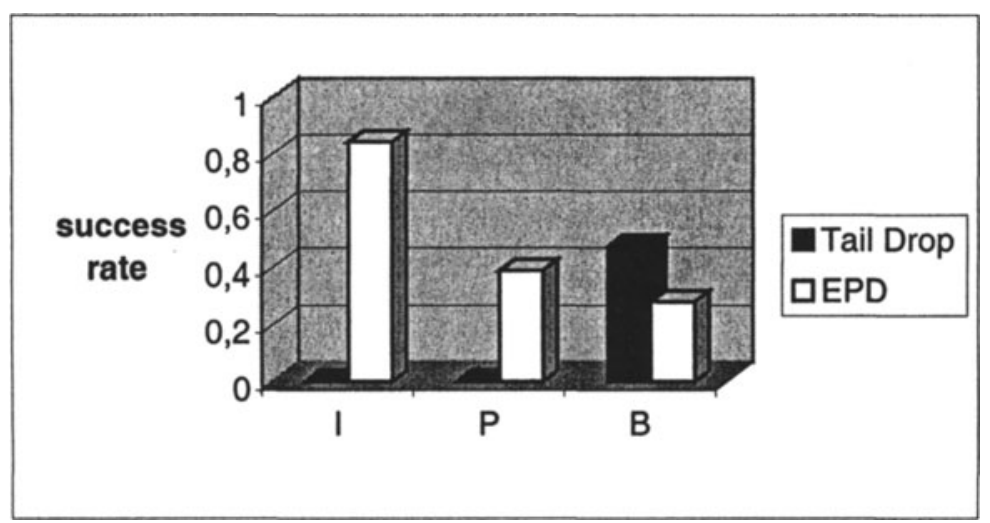

Figure 4. Picture success rate per type

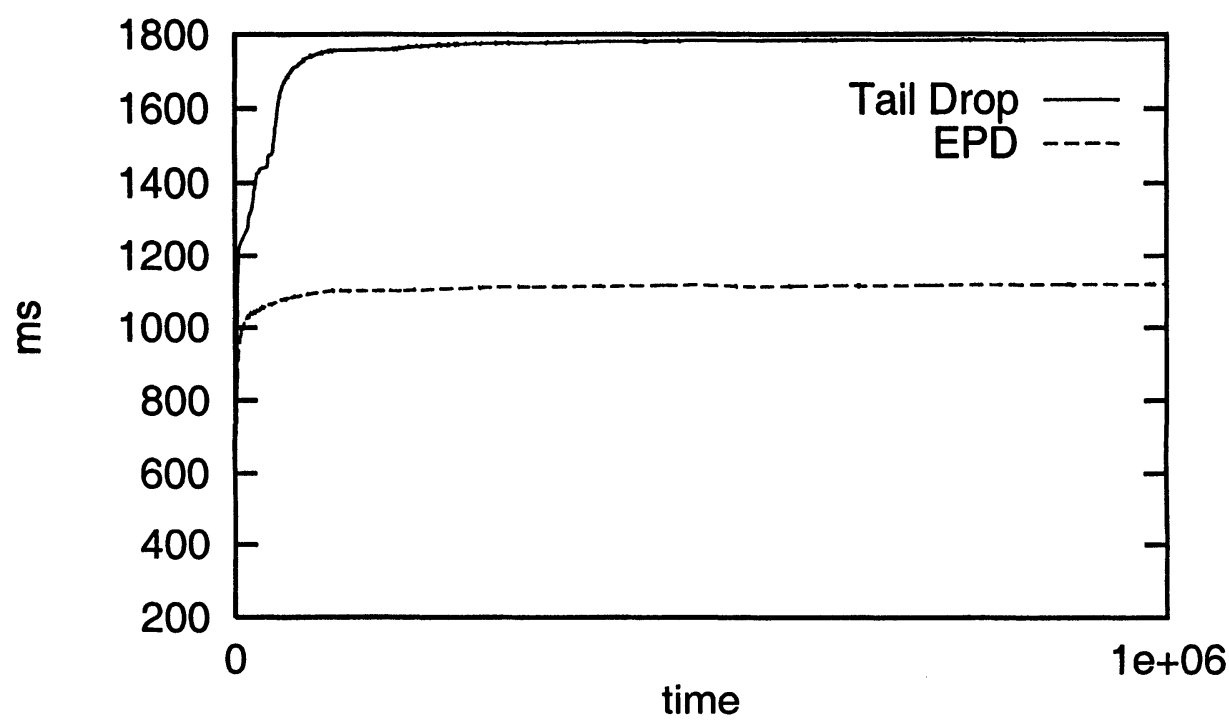

Figure 5. Picture Average delay versus time

Figure 5 presents the variation of the average transmission delay per picture over time. We remark that EPD decreases the average delay per picture. This is due to the fact that we have reduced the buffer occupation thanks to the threshold taken as a half of the buffer capacity.

\subsection{Better Early Packet Discard (BEPD)}

EPD applied to MPEG video stream with a data unit taken as a picture doesn't take into account the type nor the importance of the picture. We think that the acceptance of a picture that couldn't be decoded at the 
destination, because of the absence of its reference frame, is useless and wasteful for the resources.

We propose then, to accept in the queue only pictures that their reference frames have already been accepted or transmitted. Figure 6 presents a simulation that take into account only P or B frames that correspond to an I frame already accepted. It shows, from left to right, the success rate for Tail Drop, EPD and BEPD. We remark that BEPD improves slightly the success rate of $I$ frames, in return it decreases the number of $P$ and $B$ frames. But, these latter $P$ and B frames are obviously useless since they can't be decoded. On the other hand, we note that the number of entire GOPs has increased. This is thanks to the loss of useless $\mathrm{P}$ and $\mathrm{B}$ frames which leave the room to $\mathrm{P}$ and $\mathrm{B}$ corresponding to $\mathrm{I}$ frames already transmitted or accepted in the queue.

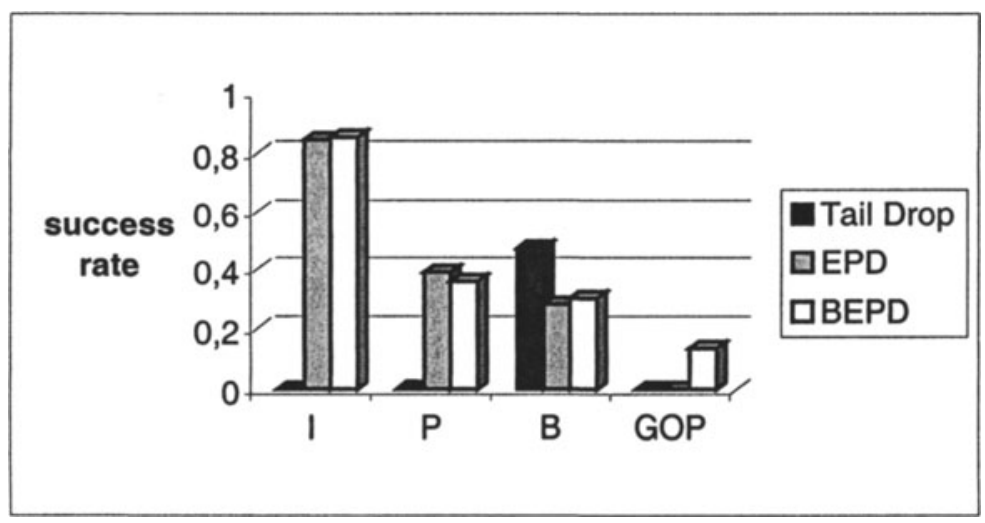

Figure 6 : success rate per type

\subsection{EPD with 3 thresholds (EPD-3)}

Performance can be significantly improved by dropping entire frames prior to congestion, as well as useless $\mathrm{P}$ and $\mathrm{B}$ frames. In addition, it is better to drop first the least important frames than others. This technique, which we call EPD-3, consists in defining 3 thresholds : $t_{B}, t_{P}, t_{I}$. As depicted in Figure 7, these thresholds define 4 modes:

- If $\mathrm{Q}<\mathrm{th}_{\mathrm{B}}$, no packet is discarded.

- If $\mathrm{th}_{\mathrm{B}}<\mathrm{Q}<\mathrm{th}_{\mathrm{P}}$, only packets belonging to a new $\mathrm{B}$ frame are discarded.

- If $\mathrm{th}_{\mathrm{P}}<\mathrm{Q}<\mathrm{th}_{\mathrm{I}}$, only packets belonging to a new $\mathrm{B}$ or $\mathrm{P}$ frame are discarded.

- If $\mathrm{Q}>\mathrm{th}_{\mathrm{I}}$, packets belonging to a new frame are discarded regardless of their type.

The goal of this technique is to privilege $I$, then $P$ and then $B$ frames. 




Figure 7 : EPD-3 queue

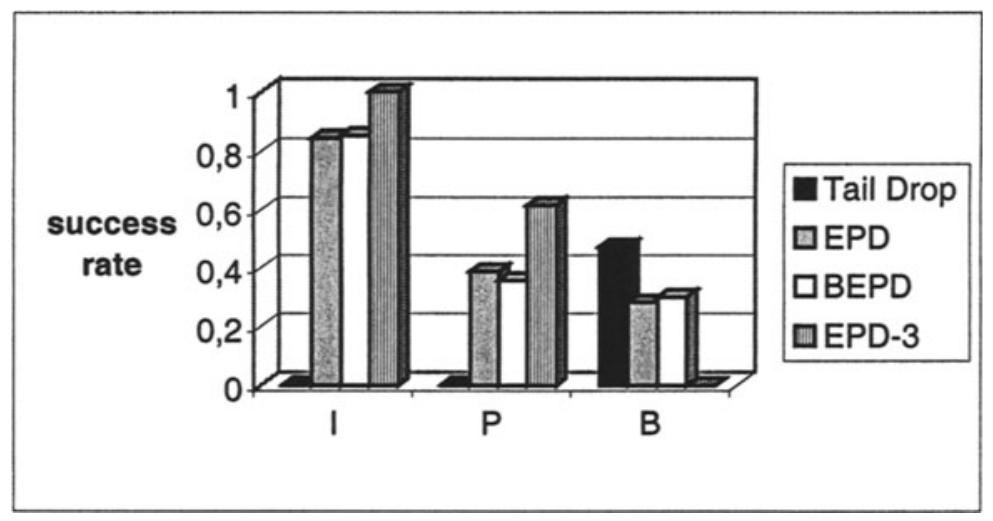

Figure 8 : Picture Success rate per type

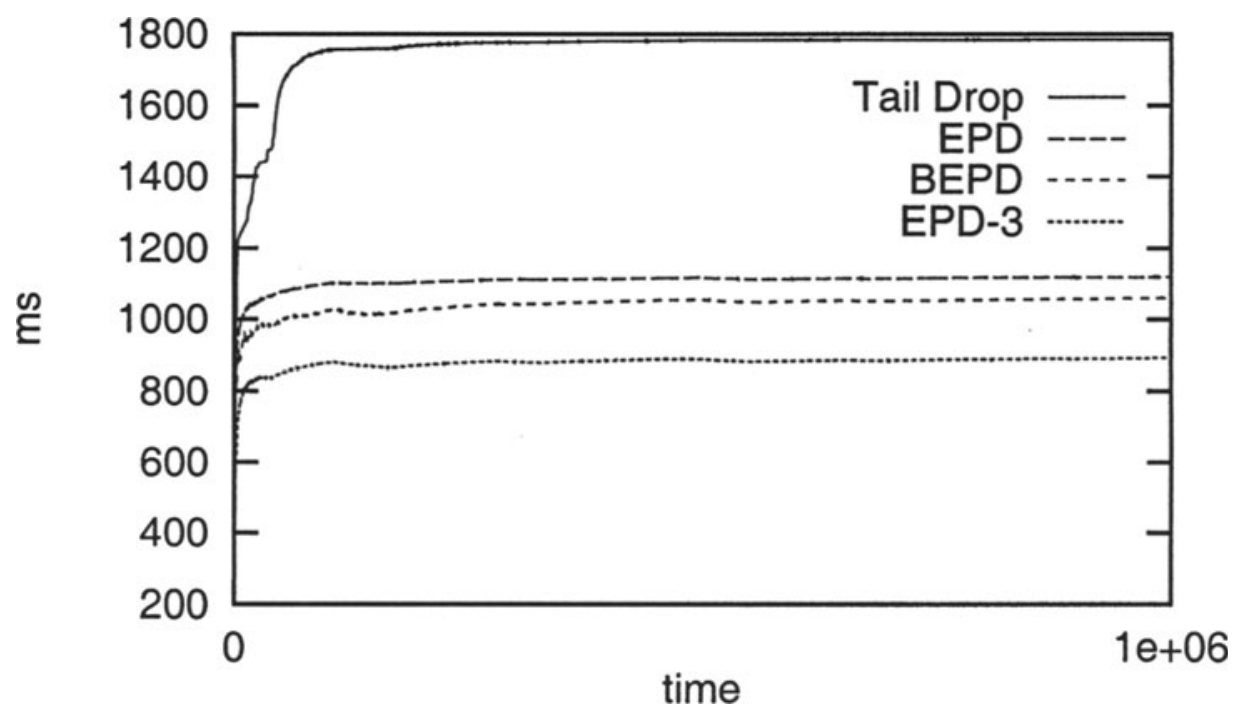

Figure 9: Picture Average delay versus time 
We run simulations with the same previous parameters and we take $\operatorname{th}_{B}=$ 20 kbytes, th $=40$ kbytes, th $_{I}=50$ kbytes.

Figure 8 shows, from left to right, the success rate per type of picture for respectively Tail Drop, EPD, BEPD and EPD-3. We note that EPD-3 protects almost all the I frames and the majority of $\mathrm{P}$ frames, which are the most important. Figure 9 shows also, that EPD-3 reduces the average delay per picture which is due to the elimination of the most frequent pictures (B frames).

\section{SPECIFICATION OF A MULTIMEDIA OPTION}

We have shown in the previous section that active queue management such as EPD or its variants (BEPD, EPD-3) improve the performance of video traffic. The deployment of such mechanisms, based on the principle of discarding a whole data unit rather than a portion of it, and/or on the type of data unit, requires that a router has the capability to delineate data units and to recognize their types.

In the DiffServ architecture, the Assured Forwarding PHB group [RFC 2597] provides delivery of IP packets in four independently forwarded AF classes. Within each AF class, an IP packet can be assigned one of three different levels of drop precedence. To introduce a priority between I, P and $B$ frames, we can use one of these AF classes with its 3 levels of drop precedence. However, to use EPD scheme and its variants, we need more information about a data unit such as the frame type, the frame number, the GOP number, etc. The DS field (6 bits as a DSCP, and 2 bits currently unused) couldn't be used to code all the information about a packet (the frame type, the frame number, the slice number, etc.) needed by EPD or its variants.

In general, video traffic is carried in the RTP transport protocol [RFC 1889] which provides end-to-end delivery services for data with real time characteristics. Those services include payload type identification, sequence numbering, time-stamping and delivery monitoring. In addition, a complete specification of RTP for a particular application requires a specific payload format that defines how a particular payload such as an audio or video encoding (MPEG1/2, H261, H263) is to be carried in RTP. Since RTP is an end-to-end protocol and its services are often integrated into the application processing, it couldn't be used by a router. So, we propose the implementation of an IP option that carries the needed information to the network level.

In IPv4, an option has the following format [RFC 791]: 


\begin{tabular}{|l|c|c|}
\hline $\begin{array}{l}\text { Option Type } \\
(1 \text { octet })\end{array}$ & $\begin{array}{c}\text { Option Length } \\
(1 \text { octet })\end{array}$ & Option data ... \\
\hline
\end{tabular}

In IPv6, an extension (equivalent to an option) has the following format [RFC 2460]:

\begin{tabular}{|l|c|l|}
\hline $\begin{array}{l}\text { Next header } \\
(1 \text { octet })\end{array}$ & $\begin{array}{c}\text { extension length } \\
(1 \text { octet })\end{array}$ & extension data ... \\
\hline
\end{tabular}

According to the RTP specification, and the information needed by active queue management, we specify the multimedia option data as follows :

\begin{tabular}{|l|l|c|}
\hline $\begin{array}{l}\text { Payload Type } \\
(7 \text { bits })\end{array}$ & $\begin{array}{l}\text { Picture type } \\
(3 \text { bits })\end{array}$ & specific payload data ... \\
\hline
\end{tabular}

Payload type : distinct payload types are assigned for different encoding media.

Picture type : defines the type of the picture (I, P, B in MPEG1/2). This field corresponds to the $P$ field defined in [RFC 2250]

EPD and its variants can be implemented with any MPEG data unit (slice, picture, GOP), so the option should hold the whole information. According to [RFC 2250] defining the RTP format for MPEG1/2 video, we specify the payload data for MPEG video as follows:

\begin{tabular}{|l|c|c|c|}
\hline $\begin{array}{l}\text { GOP number } \\
(10 \text { bits })\end{array}$ & $\begin{array}{c}\text { Picture number } \\
(10 \text { bits })\end{array}$ & $\begin{array}{c}\text { B } \\
(1 \mathrm{bit})\end{array}$ & $\begin{array}{c}\text { E } \\
(1 \mathrm{bit})\end{array}$ \\
\hline
\end{tabular}

GOP number : a sequence number incremented each time there is a new GOP (a new I picture)

Picture number : deduced from the temporal reference of the current picture within a current GOP. This value is constant for all packets of a given picture.

B : beginning of slice; the first byte of the packet data is the start of a slice $\mathrm{E}$ : end of slice; the last byte of the packet data is the end of a slice The choice of the lengths of multimedia option fields corresponds to the specification of RFC 2250. 


\section{CONCLUSION}

In this paper, we have proposed the deployment of Early Packet Discard scheme for the transmission of MPEG video streams. We have compared this mechanism to the traditional technique Tail Drop, and we have shown that EPD improves the traffic performance by decreasing the loss of reference frames (I and P). We have proposed some improvements of EPD: BEPD which accepts in the queue only frames that their reference pictures have been already accepted, and EPD-3 which in addition to the principle of EPD introduces priorities between frames using for that three thresholds. BEPD increases slightly the number of I frames and improves the quality by accepting only $\mathrm{P}$ and $\mathrm{B}$ frames which can be decoded at the destination. EPD-3 reduces further the picture delay and increases considerably the number of accepted I frames.

To deploy such mechanisms in the Internet routers, we have to carry some specific information about the flow at the network level. The DiffServ Code Point (DSCP) is not large enough to code the needed information. RTP offers all information about an encoding media, but it provides end-to-end services. So, we have proposed the implementation of an option (or extension in IPv6) that defines the characteristics of each data unit. We are inspired by the RTP payload format for MPEG1/2 to specify this option. An implementation of the option and the EPD mechanisms is carried out in a linux plateform. The tests realized with a video streaming tool have experienced the same behaviour as simulation.

\section{REFERENCES}

[MEH 98] Mehaoua, A., Boutaba, R., Iraqi, Y., "Partial versus Packet Video Discard", GlobeCom'98, Sydeney, Australia, November 1998.

[ROM 95] Romanow, A. , Floyd, S., "Dynamics of TCP Traffic over ATM Networks", in IEEE journal on selected Areas In Communications, May 1995.

[TOU 98] Tounsi, H., Toutain, L. Kamoun, F., « Best Effort Amélioré », JDIR'98 (Journées Doctorales Informatique et Réseaux), Paris, France, Avril 1998.

[TOU 99] ] Tounsi, H., Toutain, L. Kamoun, F., "Router Queue Management for Multimedia traffic in the Internet ", In International Journal on Networking Information System (NIS Journal), Journal vol2 $\mathrm{n}^{\circ} 1$ and 2, March and April 1999, Edition Hermès.

[TUR 96] Turner, J., "Maintaining high throughput during oberload in ATM switches", in Proceedings of Infocom, San Francisco, CA, April 1996. 